\title{
The ALICE TPC Readout Control Unit
}

\author{
C. González Gutiérrez, R. Campagnolo, C. Engster, A. Junique, B. Mota, L.Musa \\ CERN, 1211 Geneva 23, Switzerland \\ Carmen.Gonzalez.Gutierrez@cern.ch
}

\author{
J. Alme, J. Lien, B. Pommersche, M. Richter, K. Roed, D.Röhrich, K.Ullaland \\ University of Bergen, Norway
}

T. Alt

Ruprecht-Karls Universität Heidelberg, Physikalisches Institut, Heidelberg Germany

University of Bergen, Norway

\author{
R. Bramm \\ Institute für Kernphysik, Johann-Wolfgant-Universität, Frankfurt, Germany
}

\begin{abstract}
The Front End Electronics for the ALICE Time Projection Chamber (TPC) consists of about 560000 channels packed in 128-channel units (Front End Card). Every Front End Card (FEC) incorporates the circuits to amplify, shape, digitize, process and buffer the TPC pad signals. From the control and readout point of view the FECs are organized in 216 partitions, each being an independent system steered by one Readout Control Unit (RCU). The RCU, which is physically part of the on-detector electronics, implements the interface to the Data Acquisition (DAQ), the Trigger and Timing Circuit (TTC) and the Detector Control System (DCS). It broadcasts the trigger and clock information to the FECs, performs the initialization and readout via a high bandwidth bus, and implements monitoring and safety control functions via a dedicated $\mathrm{I}^{2} \mathrm{C}$-like link. This paper addresses the architecture and the system performance of the RCU.
\end{abstract}

\section{INTRODUCTION}

The ALICE TPC [1, 2] is readout at both end-caps by classical multi-wire proportional chambers with cathode pad readout segmented in 557568 elements. Each pad is equipped with a readout chain [3] consisting of four basic functional units: a charge sensitive shaping-amplifier, a 10-bit 25-MSPS $\mathrm{ADC}$, a pipelined digital processor and a multi-acquisition memory. The analogue functions are realized by a custom integrated circuit that incorporates 16 channels (PASA). The rest of the readout chain is integrated in a single chip, named ALTRO [4], which also implements 16 channels.
When a Level-1 trigger is received, a predefined number of samples (acquisition) are temporarily stored in a data memory. Upon Level-2 trigger arrival the latest acquisition is frozen, otherwise it will be overwritten by the next acquisition. An acquisition contains a maximum of 1000 samples per channel. The Digital Processor implements several algorithms that are used to condition and compress the signal. It produces a certain number of non-zero data packets, thus reducing the overall data volume. Each data packet is formatted with its time stamp and size information useful for the afterwards reconstruction. The output of the Data Processor is sent to a Data Memory of $4 \mathrm{Kx} 10$-bit words, able to store up to 8 acquisitions. The data is read out from the chip at speed of $40 \mathrm{MHz}$ through a 40-bit wide bus, yielding a total bandwidth of $200 \mathrm{MByte} / \mathrm{s}$.

The complete readout chain is contained in the FECs [5], which are connected to the detector by means of short capton cables. Each FEC contains 8 PASA and 8 ALTRO chips, for a total of 128 channels. A number of FECs (up to 25) are controlled by a Readout Control Unit (RCU) that implements the interface to the DAQ, the Trigger, and the Detector Control System. From the physical point of view, the two TPC readout planes are segmented in $2 \times 18$ sectors, each covering 20 degrees. Every TPC sector (15488 channels) is readout by 6 partitions (121 FECs) for a total of 216 partitions.

One of the main requirements to the RCU is set by the extremely large data volume produced by the TPC. As mentioned above, the signals produced by the TPC pads can be sampled up to 1000 times per event, thus generating about 710 MByte/event. ALICE will operate its TPC at a maximum trigger rate of $200 \mathrm{~Hz}$ in $\mathrm{Pb}-\mathrm{Pb}$ running and $1 \mathrm{kHz}$ in $\mathrm{p}-\mathrm{p}$ 
running, producing respectively 142 GByte/s and 710 GByte/s of uncompressed data. After the data compression applied in the FECs, the data volume is reduced to $20 \mathrm{GByte} / \mathrm{s}$ and 5 GByte/s respectively. Therefore, in order to be able to cope with these data throughputs, the ensemble of the RCUs has to provide a link to the DAQ with an aggregate bandwidth above 20GByte/s. This bandwidth must not decrease below $5 \mathrm{GByte} / \mathrm{s}$ in the case of high rates of small data packets, like in the p-p scenario.

The radiation load on the TPC is low $\left(1 \mathrm{krad} \oplus 10^{11}\right.$ neutrons $/ \mathrm{cm}^{2}$ over 10 years). Thus standard radiation-soft technologies are suitable for the implementation of this electronics. However, some special care should be taken to protect the system against potential damages caused by Single Event Effects.

The RCU, originally conceived and developed for the readout and control of the ALICE TPC electronics, it is being employed for the readout of other ALICE detectors (PHOS and FMD), which have a readout electronics also based on the ALTRO chip. However, the requirements in terms of bandwidth for these applications are less tight when compared to the TPC.

\section{SYSTEM ARCHITECTURE}

Although primarily developed as readout controller for the ALICE TPC FEE, the RCU has been designed to implement control, readout and monitoring functions for any front-end card based on the ALTRO chip. Therefore, while the interface to the front-end units and the core functions are tailored to the ALTRO architecture, the interface to the back-end systems (DAQ, DCS and Trigger) should be open to accommodate different technologies and possible upgrades. The resulting architecture and physical layout consist of a board based on a single FPGA, which implements all functions related to the control, configuration, readout and monitoring of the frontend units, on which can be plugged as mezzanine cards the interface boards to the specific back-end systems. In the ALICE case, these two mezzanine cards are the Detector Data Link Source Interface Unit [6] (DDL SIU), which implements a full duplex 200MByte/s optical link and is the ALICE standard interface to the DAQ, and the DCS/Trigger interface card [7], which is also an item common to a number of other ALICE detectors. In particular, the latter incorporates the LHC TTCrx chip [8], through which it receives the clock and trigger information by the TTC system, and implements an intelligent controller and DCS interface based on a FPGA with an embedded processor and an Ethernet Interface.

From the functional point of view the RCU consists of four main blocks: 1) the ALTRO Bus Controller, which implements all functions related to the communication to the front-end units during the configuration and data readout, and it is described in the sections III.A, III.B and III.C; 2) the Monitoring and Safety Module, which implements the functions described in Section III.D; 3) the interface to the DDL-SIU, which implements a bi-directional synchronous 40
$\mathrm{MHz}$ 32-bit interface protocol to transmit the trigger related data to the DAQ and can receive the configuration data for the front-end units; 4) the interface to the DCS/Trigger board implements a synchronous $40 \mathrm{MHz} 32$-bit data 16-bit address protocol to receive trigger information, configuration data for the front-end units, and transmit to the DCS the parameters relevant to monitor the behaviour of the entire front-end electronics.

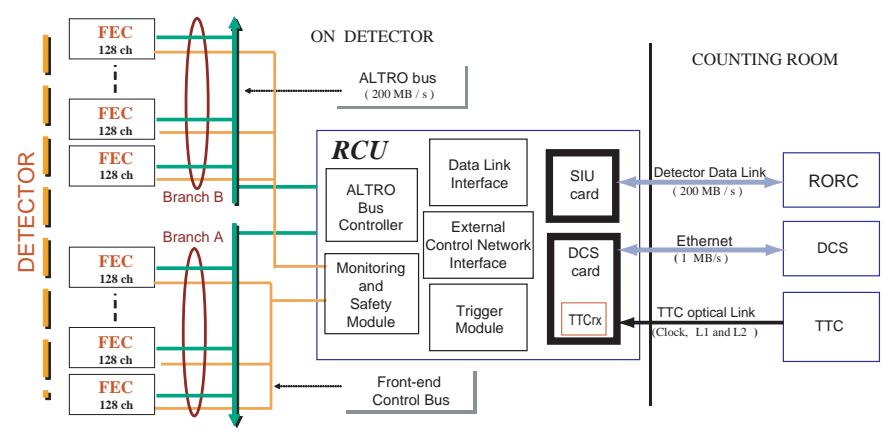

Figure 1: The architecture or the TPC readout electronics. Up to 25 FECs are interfaced via 2 separate branches of the ALTRO readout bus and the local Slow Control link, while other 2 optical links and an Ethernet connection interface the RCU respectively to the Read Out Receiver Card, the Trigger Timing Circuit and the Detector Control System.

The second part of this section focuses on the description of the two interface buses between the RCU and the front-end unit: the ALTRO bus and the Front-end Control Bus.

\section{A. ALTRO bus}

For the initialization and data readout, the communication between the RCU and the FECs is implemented via the ALTRO bus on a PCB backplane. The ALTRO bus is essentially an extension of the FEC's internal bus that allows the RCU to access the FEC's internal components. It is a multi-drop single-master bus, whereas the RCU is the MASTER unit and the FECs are the SLAVEs. In order to minimize the length of the bus lines and increase the bandwidth between FECs and RCU, the ALTRO bus Controller supports two branches running in opposite direction as it is shown in fig. 1. Electrically the bus signals are implemented as GTL lines with the exception of the sampling clock that is transmitted to the FECs as differential PECL.

The ALTRO bus implements a VME-like protocol [9] with an asynchronous handshake between the RCU and the ALTRO chips. A synchronous block transfer enhances the protocol whereas words are transferred without any acknowledgement. The bus consists of 40 bidirectional data lines for the transmission of data and address, and seven control lines. Special lines are used to distribute the trigger and clock signals. The ALTRO chip recognizes a set of Instructions. By means of these the RCU can access the 
ALTROs in the partition to write/read Configuration/Status Registers (CSR), issue Commands or read the multiacquisition memories. The ALTRO chip acknowledges the execution of any instruction. Special cases are represented by the write broadcast instruction, where all chips in the partition are concurrently addressed, and the data readout procedure, where the ALTRO acts as a master and strobes data on the RCU data FIFO (slave) synchronously without any acknowledgement. When an instruction is issued, the Command Strobe line (CSTB) must be held low until the ALTRO asserts the Acknowledge line (ACK). ACK is kept low until CSTB is de-asserted (see fig. 2 for the Read instruction and fig. 3 for the Write Instruction and Readout). Due to this handshake, the bandwidth reached during the ALTRO configuration is about $6 \mathrm{MByte} / \mathrm{s}$.

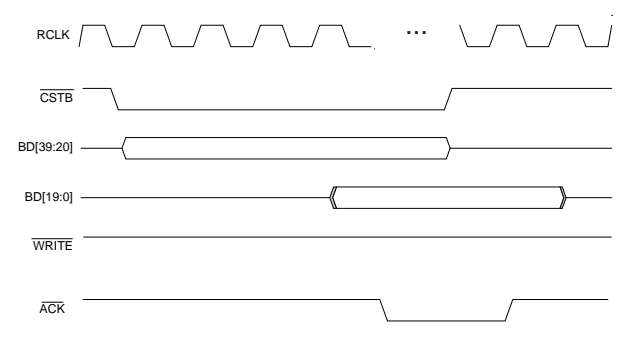

Figure 2: ALTRO bus Read instruction chronogram.

During the Readout operation all 40 data lines are used together to transfer 40bit-long words strobed by the Data Strobe signal (DSTB) with the same frequency of the system clock (40 MHz) (fig.3). The resulting bandwidth is of 200 MByte/s per branch (400 MByte/s per RCU partition).

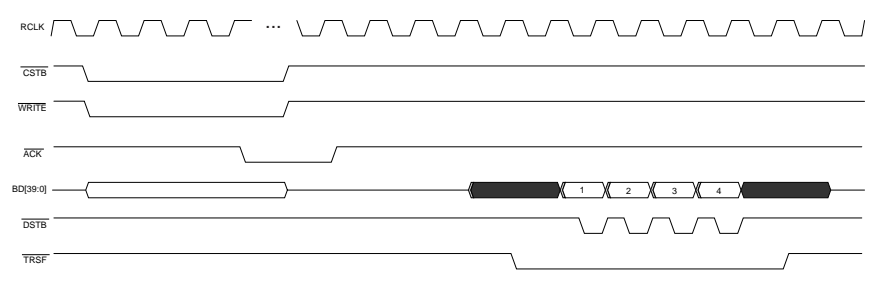

Figure 3: Readout chronogram: after the Readout instruction (write cycle) issued by the RCU, the ALTRO asserts the Transfer line (TRSF) and makes use of all 40 bit lines to transfer data words at the clock rate.

\section{B. Front-end Control Bus (FCB)}

As we have seen in the previous section, the RCU performs the configuration and readout of the FECs via the ALTRO bus. However, the FEC contains a circuit, named Board Controller (BC), implemented in a FPGA, which provides the RCU with an independent access to the FEC via a serial bus, the Front-end Control Bus. This secondary access is used to configure the FECs power state and monitor their power supplies, temperature, and the data flow. Physically, the serial bus is implemented in GTL technology as part of the ALTRO backplane.
The FCB makes use of a protocol similar to the $\mathrm{I}^{2} \mathrm{C}^{\circledR}$. The main differences consist in: 1) the use of two separate unidirectional data lines instead a single bidirectional one (SDA); 2) the data bandwidth is of $5 \mathrm{Mbit} / \mathrm{s}$ instead of the 3.4Mbit/s ( $\mathrm{I}^{2} \mathrm{C}^{\circledR}$ Ver. $2.1 \mathrm{Hs}-$ mode); 3) an additional interrupt line. The FCB begins a transaction by establishing a start condition (high to low transition of the SDA line while SCL remains high) and finishes with a stop condition (low to high transition of the SDA line while SCL remains high) (fig.4). Every transaction between the FCB master (RCU) and the slave (BC) requires the following sequence: start condition (1 clock cycle), eight bits containing the FEC's hardware address and the definition of the write or read cycle, eight bits with the address of the $\mathrm{BC}$ internal register, 16 bits with the value of the addressed registers and a stop condition (1 clock cycle). Data bytes are synchronously transferred in nine clock cycles, eight cycles for the data bits plus one for the acknowledge information sent back from the target unit.

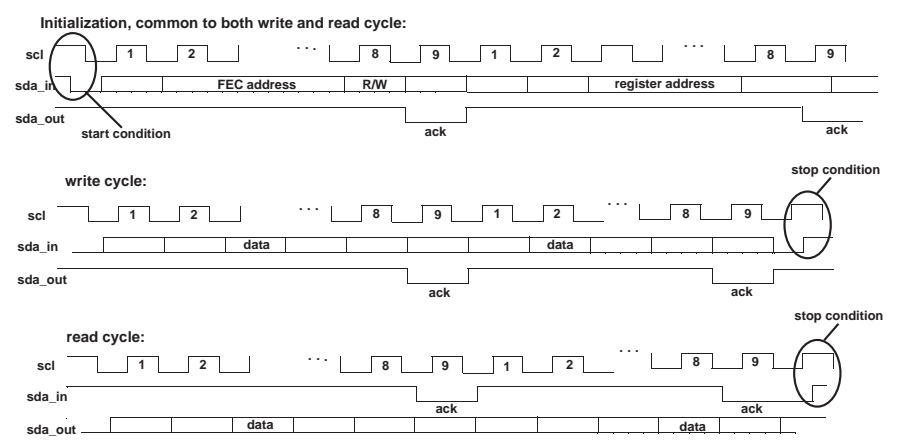

Figure 4: Front-end Control Bus protocol. The standard $I^{2} C^{\circledR} \mathrm{SDA}$ line is split in 2 unidirectional lines: SDA_in from the RCU to the FECs and SDA_out in the opposite direction.

\section{RCU FUNCTIONS}

The RCU performs key system-level functions: distribution of Trigger and Clock signals to the FECs, configuration of each front-end channel, readout of trigger related data from the FECs, subsequent formatting and transfer to the DAQ system. In parallel, the RCU is also responsible for monitoring the voltages, currents, temperature and a number of functional parameters. In case any of the monitored quantities shows an anomalous behaviour, the RCU takes the appropriate action in order to prevent any damage to the FECs or severe system errors.

\section{A. Trigger and Clock Distribution}

The trigger information and the LHC clock are transmitted to the RCU via the TTCrx chip of the DCS board. The incoming clock (RCLK) and a fixed latency Level-1 trigger pulse, originated in the A-channel of the TTCrx, are broadcasted to all FECs through the ALTRO bus. The sampling clock (SCLK) is derived from the RCLK through a PLL. Its value, which ranges from 5 to $10 \mathrm{MHz}$, is distributed to the FECs via a differential line in order to minimize the 
noise and, therefore, the signal jitter. The Level-1 trigger word (10 bits) and Level-2 trigger messages (several bytes of information) are received from the TTCrx B-channel. The Level-1 word and part of the Level-2 message are copied in the event data packet header shipped to the DAQ. The Level-2 message is used to compare the LHC bunch crossing number with the local one, and to generate the Level-2 accept/reject signals for the FECs. Three trigger running modes are possible in the RCU: software trigger mode, used for debugging and calibration purposes; Level-1 mode for detectors using a single-level trigger; and Level-1 + Level-2 mode as in the standard running mode foreseen for the ALICE TPC. In the case of the Level-1 mode, the RCU self generates the Level-2 after the ALTRO acquisition window.

\section{B. Configuration of the front-end units}

The configuration parameters for the ALTROs and BCs are stored in the RCU Instruction Memory through one of the following two interfaces: the DCS or the DDL. A sequencer fetches these instructions, decodes their content and executes them in the form of a single ALTRO instruction (RCU micro instruction) or sequence of them (RCU macro-instruction). The macro instructions allow, for example, to write or to verify the full content (or part of it) of a given ALTRO pedestal memory. Another example is a RCU macro that handles a complete trigger sequence to exercise the readout chain. Due to the large configuration data volume, the predicted flux of particles for the ALICE TPC leads to one Single Event Upset in the configuration parameters per run (4 hours). Given the structure of the LHC beam, it is however possible to use the orbit gap $(88 \mu \mathrm{s})$ to check and correct the configuration. The verification of the complete configuration can be repeated with a period of $5 \mathrm{~s}$.

\section{Data Readout}

The readout of one event is performed in two separate phases, which are consecutive for a given event, but can otherwise be activated concurrently. In a first phase the trigger information is received by the RCU and broadcast to all modules in the subsystem, starting the digitisation of each channel, which lasts for $88 \mu$ s (TPC drift time). During this phase data processing takes place in the ALTROs. In the second phase, information is moved by the RCU from the ALTRO's multi-event buffer to the DAQ, on a channel-bychannel basis. The time needed to complete the second phase depends on the size of the event, but other triggers can be processed during the readout of the previous event, as long as the multi-event buffers in the FEC are not full. Dead time can be generated only when this condition occurs. FECs containing valid data are enabled to assert data on the bus by individual addressing, according to an Active Channel List stored in the RCU. A readout time optimization can be obtained by an additional exclusion from the readout list of all empty channels for that specific event. This information is sent by each FEC's BC to the RCU after the L2-accept. With a higher granularity, an Active Front-End Card List addresses only the FECs participating to the readout, skipping the inactive ones.

The readout is performed concurrently on the two ALTRO bus branches. The incoming event data is stored in two memories, working in interleaved mode, each being able to store the event data of one single ALTRO channel. While data from one memory is being pushed into the DDL, data from the next ALTRO channel is stored into the other one. The 40bit wide ALTRO data packets are formatted into 32-bit wide packets and wrapped in the ALICE DDL data format, with 7 headers and one trailer word. A further option sets the channel readout order following the detector topology, in order to facilitate the on-line data processing. The full trigger distribution scheme and the readout chain is depicted in fig. 5 .

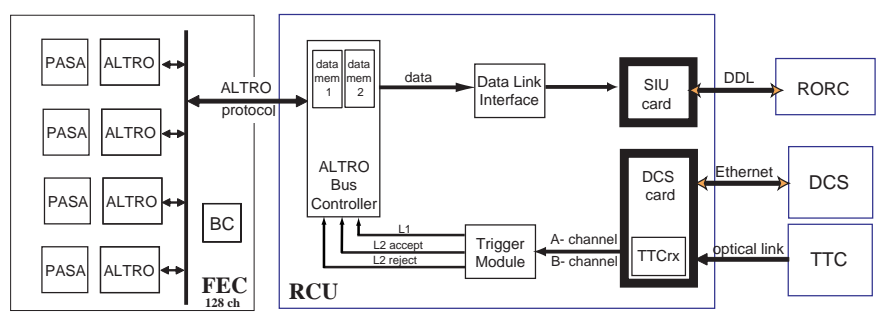

Figure 5: FEE trigger distribution and readout scheme.

\section{Safety and monitoring}

The RCU controls the power state, voltages, currents and temperature of the FECs. In detail, every FEC contains a 10bit, 5-channel ADC with an on-chip temperature sensor and an $\mathrm{I}^{2} \mathrm{C}^{\circledR}$ interface. One channel is reserved to read the temperature, while the other 4 are fed with 2 voltages and 2 currents (analogue and digital) measured at the input of the FEC. The $\mathrm{BC}$ logic includes an $\mathrm{I}^{2} \mathrm{C}^{\circledR}$ master to read this ADC. The communication between the two units is synchronized by a clock signal with a frequency of $150 \mathrm{KHz}$. At this rate, every $2 \mathrm{~ms}$ the $\mathrm{BC}$ reads the 5 parameters and updates the corresponding registers. In addition, the $\mathrm{BC}$ contains the configuration, status and error registers, and a set of counters that measure a number of critical signals (e.g. the Level-1 and Level-2). The table is accessible both via the ALTRO bus (during the configuration phase) and the FCB (in the configuration phase and data taking phase).

At power-up, the RCU downloads into the $\mathrm{BC}$ the reference range for the monitored quantities. As soon as one of these parameters goes out of range, the BC asserts the interrupt. The RCU starts polling the error register of each FEC to identify the error source. In the event of an hard error (temperature or currents over thresholds, voltage under thresholds, card power regulators error), the RCU switches off immediately the corresponding FEC. Immediately after a recovery procedure will be executed to diagnose the occurred error and report to the DCS system. It is important to note that, during these operations, the communication to the other cards of the branch is not perturbed. 


\section{RCU PHYSICAL DESCRIPTION}

The RCU is a $22.5 \times 15 \mathrm{~cm}^{2}$ double-side board. With reference to fig. 6 , top side contains two groups of connectors, one group per branch, the GTL transceivers, an ALTERA FPGA (APEX20K), and some auxiliary minor component. The bottom side, apart the power supplies for the board and for the termination of the backplane GTL lines, it only contains the connectors for the DDL-SIU board (CMC format) and for the DCS card (DIMM).

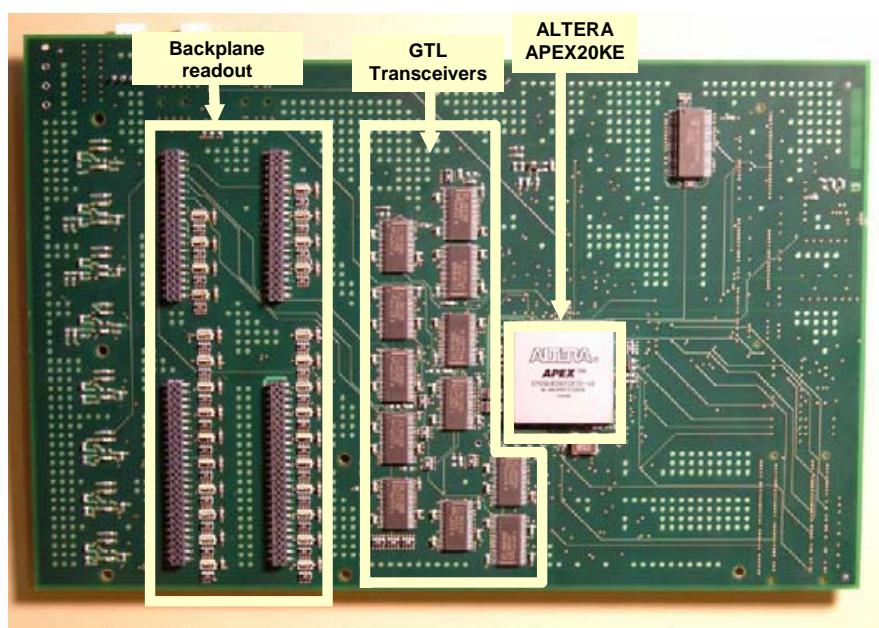

Figure 6: RCU Mother board. On the board back side the connectors for the mezzanine cards.

\section{SYSTEM PERFORMANCE}

The largest readout partition consists of 3200 ALTRO channels (25 FECs). A typical configuration for such a partition consists of about $350 \mathrm{Kbytes}$, and can be as large as 7 MBytes worst case (1000 10-bit pedestal values per channel). As described above, configuration data can be transferred to the RCU both via the DDL and the DCS. In both cases the data transfer takes less than $50 \mathrm{~ms}$ for the typical configuration. The subsequent transfer to the FECs requires about $150 \mathrm{~ms}$. As far as the readout performance is concerned, the ALTRO bus has a measured bandwidth of $260 \mathrm{MByte} / \mathrm{s}$ and 55MByte/s for respectively the $\mathrm{Pb}-\mathrm{Pb}$ and $\mathrm{p}-\mathrm{p}$ running conditions. In the former case the average data block size is of $200 \mathrm{Byte} / \mathrm{channel}$; in the latter is $20 \mathrm{Byte} /$ channel.

The Local Slow Control network runs at a clock frequency of $5 \mathrm{MHz}$. However, its protocol requires the transmission of a large number of control words. A single 16-data bit transaction, e.g., requires $8 \mu \mathrm{s}$. When an interrupt occurs, the RCU starts polling the error/status register of all FECs of one branch. This action could require up to $100 \mu$ s in the case of a readout partition with $13 \mathrm{FEC} / \mathrm{branch}$.

\section{CONCLUSIONS}

The RCU, which is a key unit of the ALICE TPC FEE, is a board based on a single FPGA, which implements the control, readout and monitoring of the ALICE front-end cards based on the ALTRO chip. In the ALICE application it is equipped with two mezzanine cards, which implement the interface to the DAQ, and to the Trigger and DCS systems. The RCU fulfils the ALICE requirements providing an aggregate bandwidth between FECs and DDLs in excess of 34 GByte/s for the $\mathrm{Pb}-\mathrm{Pb}$ running conditions, and of about 12 GByte/s in the p-p scenario.

Two prototypes have been successfully tested with a sizeable fraction of the final complete system (two complete readout partitions) at the PS test beam. Presently the RCU is being finalized for mass production in 2005 .

\section{REFERENCES}

[1] ALICE Collaboration, A Large Ion Collider Experiment, ALICE TPC - Technical Report, ISBN 92-9083-155-3, December 1999, CERN, Geneve, Switzerland.

[2] L. Musa, The Time Projection Chamber for the ALICE Experiment, $16^{\text {th }}$ International Conference on Ultrarelativistic Nucleus-Nucleus Collisions: Quark Matter 2002, Nantes, France, July 2002. Nuclear Physics A715 (2003) 843c-848c

[3] L. Musa et al., The ALICE TPC Front End Electronics, Proc. of the IEEE Nuclear Science Symposium, October 2003, Portland

[4] R. Esteve Bosch et al., The ALTRO Chip: A 16-channel $A / D$ Converter and Digital Processor for Gas Detectors, IEEE Transaction on Nuclear Science, Vol. 50 No. 6, December 2003.

[5] R. Campagnolo et al., Performance of the ALICE TPC Front End Card, Proc. of the $9^{\text {th }}$ Workshop on Electronics for LHC Experiments, Amsterdam, September - October 2003.

[6] ALICE DATA Acquisition, http://aldwww.cern.ch

[7] The DCS Board Specifications, http://www.kip.uniheidelberg.de/ti/DCS-Board/current/

[8] J. Christiansen, et al., Receiver ASIC for Timing, Trigger and Control Distribution in LHC Experiments, IEEE Trans. Nuclear Science, Vol. 43, June 1996, pp. 1773-1777

[9] L. Musa et al., The ALICE TPC Readout chip, http://eped-alice-tpc.web.cern.ch/ep-ed-alice-tpc/altro_chip.htm 